\title{
Exploring the connection between coronal and footpoint sources in a thin-thick target solar flare model
}

\author{
M. Battaglia and A. O. Benz \\ Institute of Astronomy, ETH Zurich, 8092 Zurich, Switzerland \\ e-mail: battaglia@astro.phys.ethz.ch \\ Received 22 January 2007 / Accepted 8 February 2007 \\ ABSTRACT

\begin{abstract}
Context. Hard X-ray emission of coronal sources in solar flares has been observed and studied since its discovery in Yohkoh observations. Several models have been proposed to explain the physical mechanisms causing this emission and the relations between those sources and simultaneously observed footpoint sources.

Aims. We investigate and test one of the models (intermediate thin-thick target model) developed on the basis of Yohkoh observations. The model makes precise predictions on the shape of coronal and footpoint spectra and the relations between them, that can be tested with new instruments such as RHESSI.

Methods. RHESSI observations of well observed events are studied in imaging and spectroscopy and compared to the predictions from the intermediate thin-thick target model.

Results. The results indicate that such a simple model cannot account for the observed relations between the non-thermal spectra of coronal and footpoint sources. Including non-collisional energy loss of the electrons in the flare loop due to an electric field can solve most of the inconsistencies.
\end{abstract}

Key words. Sun: flares - Sun: X-rays, $\gamma$-rays - acceleration of particles

\section{Introduction}

How well do we understand the physics behind solar flares? Solar flares release a large portion of their energy within seconds to minutes. A significant part of this energy goes into accelerated electrons and ions which then precipitate to the chromosphere along the field lines of a magnetic loop. The chromosphere acts as a thick target on the precipitating particles, leading to characteristic hard X-ray (HXR) emission. The first observations of such footpoint sources were made by Hoyng et al. (1981). Masuda et al. (1994) found another HXR source near the top of flare loops in Yohkoh observations. Feldman et al. (1994) analyzed such coronal sources with Yohkoh BCS and SXT. Due to the normally low coronal densities one would expect a thin target emission from this coronal source. However, the observations by Feldman et al. (1994) yield quite high column densities $\left(10^{20} \mathrm{~cm}^{-2}<N<7 \times 10^{20} \mathrm{~cm}^{-2}\right)$ in the coronal source that would act as a partly thick target on electrons below a certain energy. On the basis of the observations by Masuda et al. (1994) and Feldman et al. (1994), Wheatland \& Melrose (1995) developed a simple model that has been used and investigated further (e.g. Metcalf \& Alexander 1999; Fletcher \& Martens 1998). The model consists of four basic elements; a particle accelerator on top of a magnetic loop, a coronal source visible in SXR and HXR, collision-less propagation of particles along the magnetic loop and HXR-footpoints in the chromosphere. The coronal source acts as an intermediate thin-thick target on electrons depending on energy (thick target for lower energetic electrons, thin target on higher energies). We will therefore refer to this model as intermediate thin-thick target, or ITTT model.
For large enough column depths or steep enough electron spectra, almost all electrons would be stopped in the loop and no footpoints could be observed. Such events were observed with Yohkoh and later with RHESSI. Veronig \& Brown (2004) analyzed flares with faint footpoint emission but a dense loop acting as a thick target on electrons of energies up to $60 \mathrm{keV}$.

Due to its high spectral and spatial resolution, RHESSI (Lin et al. 2002) provides the means to perform imaging spectroscopy on events with multiple sources. Battaglia \& Benz (2006) showed that it is possible to study the non-thermal X-ray emission of coronal sources and footpoints and the relations between them in detail.

Therefore, the ITTT model and its predictions can be tested using RHESSI data of well observed events. It is the simplest model that can explain the Yohkoh data. Can it also explain RHESSI data?

\section{Theoretical model}

The ITTT model features a dense coronal source into which a beam of electrons with a power-law energy distribution is injected. Some high energy electrons then leave the dense region, precipitating down to the chromosphere. The coronal region acts as a thick target on particles with energy lower than a critical energy $E_{\mathrm{c}}$ and as thin target on electrons with energy larger than the critical energy. This results in a characteristic HXR spectrum as well as SXR emission due to collisional heating of the coronal region. The altered electron beam reaches the chromosphere, causing thick target emission in the footpoints of the magnetic loop. The predicted photon spectra are shown in Fig. 1. The coronal spectrum consists of two power-law components with a 


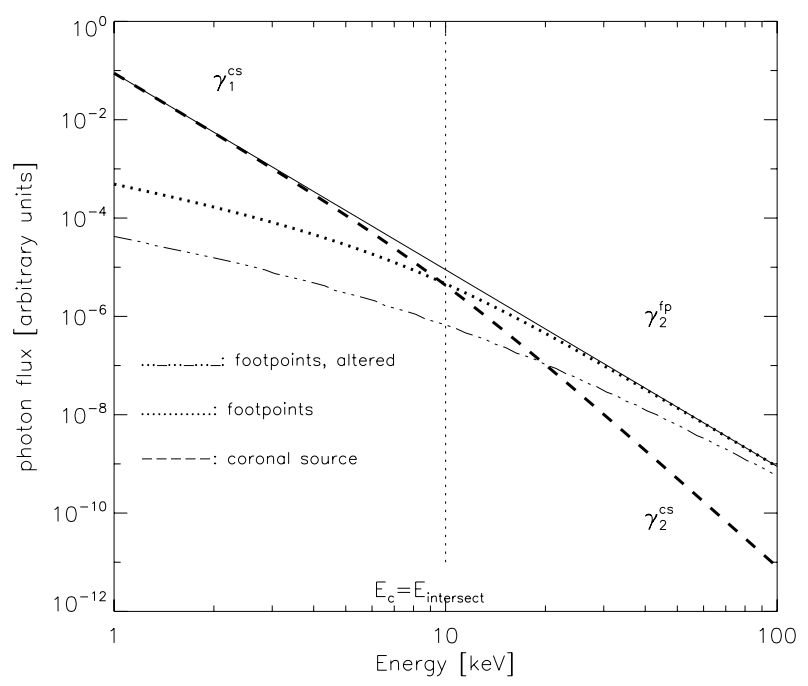

Fig. 1. Schematic photon spectra for electron distribution with powerlaw spectral index $\delta=5$ and critical energy $E_{\mathrm{c}}=10 \mathrm{keV}$. The dashed line gives the spectrum of the coronal source with the spectral indices $\gamma_{1}^{\mathrm{cs}}=\gamma_{2}^{\mathrm{fp}}=\delta-1$ and $\gamma_{2}^{\mathrm{cs}}=\delta+1$. The dotted line indicates the combined spectrum of the two footpoints. The footpoint spectrum is a power-law with spectral index $\gamma_{2}^{\mathrm{fp}}=\delta-1$ at high energies. The solid line is the total of coronal source and footpoints (pure thick target). The dash-dotted line gives the footpoint spectrum from an electron population that has been altered in the loop (see Discussion).

break at $E_{\mathrm{c}}$. The footpoint spectrum has a break at the same energy as the coronal spectrum and is a power-law at high energies. The model predicts the following properties:

1. The coronal and footpoint spectra intersect at the critical en$\operatorname{ergy}\left(E_{\mathrm{c}}=E_{\text {intersect }}\right)$.

2. There is a break at $E_{\mathrm{c}}$ in the individual spectra of coronal source and footpoints, but not in the sum of the two spectra.

3. The spectral indices below and above the break in the coronal source have a difference of $\gamma_{2}^{\mathrm{cs}}-\gamma_{1}^{\mathrm{cs}}=2$.

4. The spectral index of the coronal source below the break is equal to the spectral index of the footpoints above the break $\left(\gamma_{1}^{\mathrm{cs}}=\gamma_{2}^{\mathrm{fp}}\right)$.

5. The difference between the spectral indices of the coronal source and the footpoints at high energies is $\gamma_{2}^{\mathrm{cs}}-\gamma_{2}^{\mathrm{fp}}=2$.

6. Coronal source and footpoints have the same intensity at $E_{\mathrm{c}}$.

7. SXR and HXR emission in the coronal source are spatially coincident.

\section{Observations}

\subsection{Event selection and spectral analysis}

We analyzed the five events described in Battaglia \& Benz (2006) in an interval of 3 RHESSI spin-periods (about 12 s) around the time of maximum HXR-emission. The events are listed in Table 1.

Imaging spectroscopy applying the Clean algorithm for image reproduction was used to produce spectra of coronal sources and footpoints. Reasons for favoring Clean over Pixon are discussed in Battaglia \& Benz (2006). To compare the observations with the model, the footpoints where treated as one region and only a total spectrum over both footpoints has been computed. The coronal sources have been fitted with a thermal component and a non-thermal power-law, the footpoints only with a nonthermal component. Varying the starting fit parameters and the
Table 1. Eventlist. The time indicates the peak time in the $50-100 \mathrm{keV}$ energy band.

\begin{tabular}{lccr}
\hline \hline No. & Date & Time & GOES class \\
\hline 1 & 4-Dec.-2002 & $22: 47: 02$ & M2.7 \\
2 & 24-Oct.-2003 & $02: 48: 42$ & M7.7 \\
3 & 1-Nov.-2003 & $22: 33: 14$ & M3.3 \\
4 & 13-Jul.-2005 & $14: 14: 30$ & M5.1 \\
5 & 30-Jul.-2005 & $06: 32: 06$ & X1.3 \\
\hline
\end{tabular}

fit energy range provided a validation for the stability of the fit and an estimate of the range of spectral index values. The spectra and fits are shown in Fig. 2.

\subsection{Density and critical energy}

In the ITTT model, the critical electron energy for the transition between thick and thin target for a given column density $N_{0}$ is given by equating the source size to half of the mean free path,

$E_{\mathrm{c}}=\sqrt{2 K N_{0}}$

with the constant $K=2 \pi \mathrm{e}^{4} \ln \Lambda$. The Coulomb logarithm $\ln \Lambda$ is about 20 for the electron densities and temperatures in the presented sample. The column depth the electron beam passes in the coronal source was computed using RHESSI and GOES data, following the treatment in Wheatland \& Melrose (1995). For a source volume $V$, a total source diameter of $V^{1 / 3}$ is assumed. An electron beam injected in the middle of the source would then travel half this distance, giving a column depth of:

$N_{o}=\frac{n_{\mathrm{e}} V^{1 / 3}}{2}=\sqrt{E M} \cdot \frac{A^{-1 / 4}}{2}$

where the electron density $n_{e}=\sqrt{E M / V}$ using the observed emission measure EM and the volume $V=A^{3 / 2}$. The area $\mathrm{A}$ was measured from the $50 \%$ contour of the coronal source in RHESSI Clean images at energy $10-12 \mathrm{keV}$, taking into account the Clean-Beam size and effects of the pixel size on the contour determination.

The emission measure was computed from spectral fits to RHESSI full sun spectra and the spectrum of the coronal source only. As noted by Battaglia \& Benz (2006), the thermal footpoint emission generally is very faint in RHESSI observations. Therefore, the thermal emission measured in full sun spectra is predominantly coronal emission. However, the spectral fittings deviate slightly, the temperature being lower and the emission measure higher for the imaging spectroscopy fit. We therefore use both as a range of possible column densities.

Additionally, we computed the GOES emission measure and temperature for comparison. The range of all RHESSI and GOES emission measures then gives an estimate of the accuracy for the column densities and critical energies (Table 2).

\subsection{Position of coronal source at different energies}

The ITTT model predicts spatial coincidence of the coronal emission for all energies. To check for this, Clean images at different energies where made and the centroid of the $50 \%$ emission of the coronal source was computed in order to get the position. At energies higher than $20 \mathrm{keV}$, the footpoint emission starts to dominate, making an accurate determination of the coronal source-position difficult. We determined the centroid positions for the energy range 6-12 keV (thermal) and 16-22 keV (partially non-thermal). 

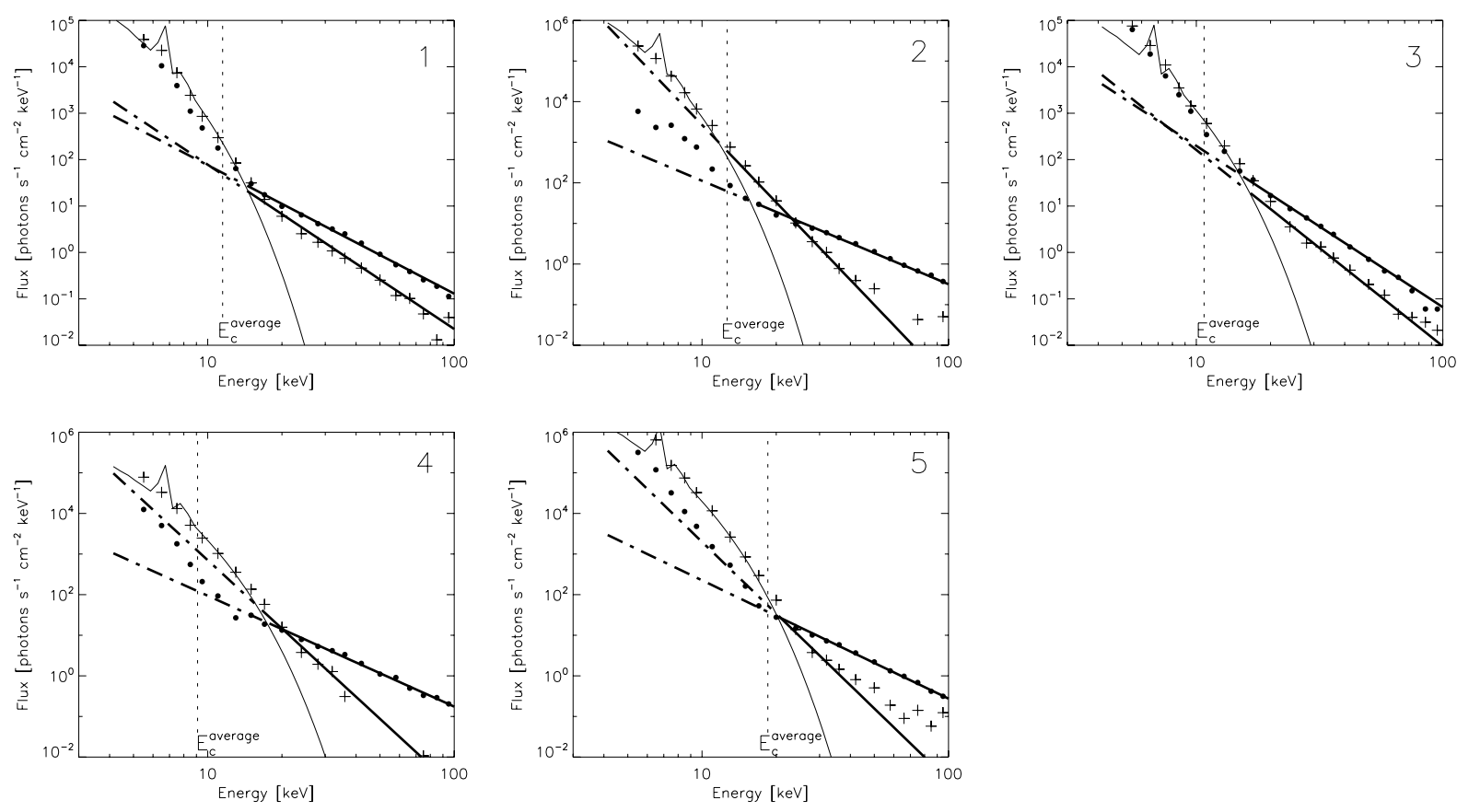

Fig. 2. Coronal (crosses) and footpoint (dots) spectra of the five analyzed events. The thin solid line indicates the thermal fit to the coronal source. The thick solid lines give the power-law fits to coronal source and footpoints. Their extension to low energies (not observed) is given as a dot-dashed line. The dotted line indicates the average critical energy.

Table 2. Summary of observed parameters to be compared with the theory. The power-law spectrum of the coronal source can only be observed at energies higher than the thermal emission, therefore no value for the part below the break could be determined.

\begin{tabular}{lccccc}
\hline \hline Event & 1 & 2 & 3 & 4 & 5 \\
\hline$\gamma_{1}^{\mathrm{cs}}$ & - & $(6.3 \pm 0.1)$ & - & - & - \\
$\gamma_{2}^{\mathrm{cs}}$ & $3.5 \pm 0.7$ & $6.3 \pm 0.1$ & $4.2 \pm 0.4$ & $5.6 \pm 0.2$ & $5.9 \pm 1.0$ \\
$\gamma_{2}^{\mathrm{pp}}$ & $2.8 \pm 0.2$ & $2.5 \pm 0.1$ & $3.5 \pm 0.1$ & $2.7 \pm 0.2$ & $2.9 \pm 0.1$ \\
$\gamma_{2}^{\mathrm{cs}}-\gamma_{2}^{\mathrm{fp}}$ & $0.7 \pm 0.7$ & $3.8 \pm 0.1$ & $0.7 \pm 0.4$ & $2.9 \pm 0.3$ & $3 \pm 1.0$ \\
$N_{0}\left(\mathrm{~cm}^{-2}\right)$ & $(2.5-4.9) \times 10^{19}$ & $(2.8-6.1) \times 10^{19}$ & $(2.1-4.3) \times 10^{19}$ & $(2.6-3.0) \times 10^{19}$ & $(7.1-12) \times 10^{19}$ \\
$E_{\mathrm{c}}(\mathrm{keV})$ & $9.5-13.5$ & $10.3-15$ & $8.9-12.6$ & $7.7-10.5$ & $16.2-20.8$ \\
$E_{\text {intersect }}(\mathrm{keV})$ & 10.3 & 23.1 & 7.6 & 20.2 & 20.9 \\
\hline
\end{tabular}

\section{Results}

The main numerical results are given in Table 2 . We find column densities between $2.1 \times 10^{19}<N_{0}<1.2 \times 10^{20} \mathrm{~cm}^{-2}$. This yields a range of $E_{\mathrm{c}}$ from $7.7 \mathrm{keV}-20.8 \mathrm{keV}$, significantly lower than the values calculated by Wheatland \& Melrose for Yohkoh events $\left(15 \mathrm{keV}<E_{\mathrm{c}}<40 \mathrm{keV}\right)$. The comparison of the data with the predictions from the ITTT model is not always possible due to the presence of the thermal component. Especially, the estimated critical energy is within the range dominated by the thermal component in all events. Comparing the data with the predictions from the ITTT model gives the following results, following the numbering in Sect. 2:

1. The power-law fits of the non-thermal coronal and footpoint spectra intersect at energies $E_{\text {intersect }}=7.6-23.1 \mathrm{keV}$. In 3 events, this energy is within or just outside the estimate for the critical energy derived from Eqs. (1) and (2). Therefore $E_{\mathrm{c}} \approx E_{\text {intersect. In two events (Nos. } 2}$ and 4$), E_{\text {intersect }}$ is significantly higher than the computed $E_{\mathrm{c}}\left(E_{\text {intersect }}>E_{\mathrm{c}}\right)$.

2. In the events with $E_{\mathrm{c}} \approx E_{\text {intersect }}$ the estimated break energies and intersection energies are in the energy range dominated by the thermal emission of the coronal source. A break in the coronal spectrum can therefore not be detected, but might still exist. In events Nos. 2 and 4 , where $E_{\text {intersect }}>E_{\mathrm{c}}$, the intersection point of the power-law spectra is observed, but without a break, contradicting the prediction.

3 . In event 2 , the intersection energy is well outside the thermal emission but $\gamma_{2}^{\mathrm{cs}}-\gamma_{1}^{\mathrm{cs}} \neq 2$ in the coronal source, contrary to the prediction.

4. In event $2, \gamma_{1}^{\mathrm{cs}}>\gamma_{2}^{\mathrm{fp}}$, not equal as expected from the model.

5. $\gamma_{2}^{\mathrm{cs}}-\gamma_{2}^{\mathrm{fp}}>2$ in three events and $\gamma_{2}^{\mathrm{cs}}-\gamma_{2}^{\mathrm{fp}}<2$ in two events.

6. In three events, $E_{\mathrm{c}}$ and $E_{\text {intersect }}$ of the non-thermal fits are within the thermal emission of the coronal source. In event 2 , $E_{\text {intersect }}$ is observed with the coronal source being brighter than the footpoints at $E_{\mathrm{c}}$.

7. No significant positional differences between energies 6-12 keV and 16-22 keV have been found (see Fig. 3). Due to the increasing footpoint brightness at higher energies, an accurate determination of the coronal source centroid above $22 \mathrm{keV}$ is not possible.

\subsection{Energy input into the corona}

Wheatland \& Melrose (1995) proposed that the collisional energy necessary to produce the non-thermal bremsstrahlung emission in the coronal source is sufficient to account for the observed 


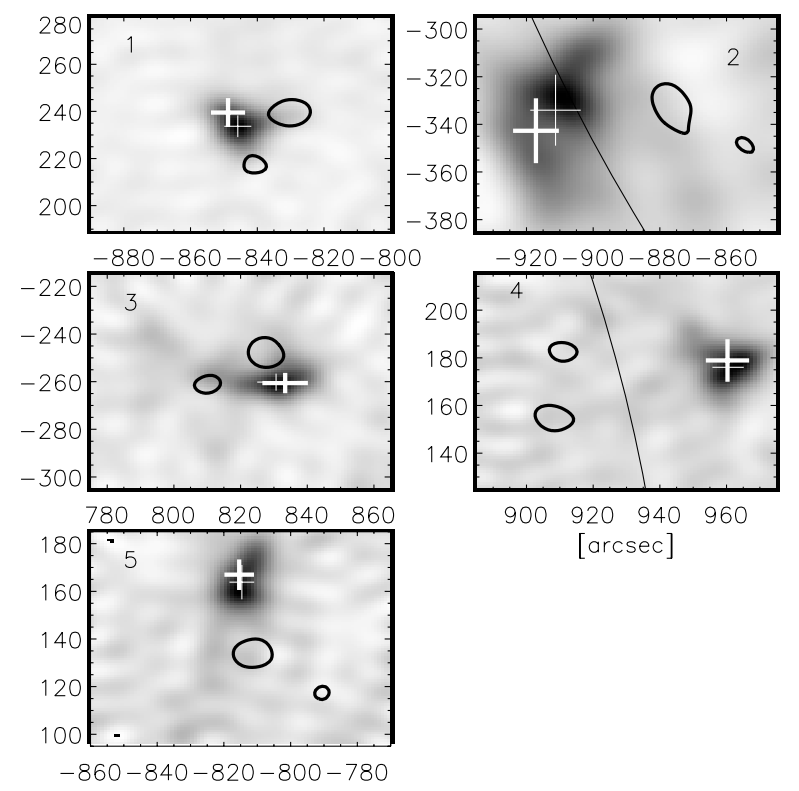

Fig. 3. Images of all events at energies $10-12 \mathrm{keV}$. The $50 \%$ contours of the maximum of the footpoints are given (black). The white crosses indicate the position of the $50 \%$ centroid at energies $6-12 \mathrm{keV}$ (thin) and $16-22 \mathrm{keV}$ (thick) with error bars.

heating of the coronal source. They referred to standard flare values (flux, duration, electron spectral index) to estimate the nonthermal energies. With the observations presented here the energy input into the individual coronal source can be determined.

We compute the change in thermal energy $\Delta E_{\text {th }}$ in the coronal source between two times in the flare. The thermal energy is calculated according to $E_{\text {therm }}=3 k_{\mathrm{B}} T \sqrt{E M \cdot V}$ with a filling factor of unity.

The non-thermal energy input in the coronal source is computed from the non-thermal coronal spectrum. As cutoff energy, we used the intersection point of the thermal and nonthermal emission. Saint-Hilaire \& Benz (2005) found that the average relation between photon-turnover and electron-cutoff is $E_{\text {cutoff }}^{\text {electron }}=E_{\text {turnover }}^{\text {photon }} \cdot 1.7$. This gives an upper limit for the electron cutoff energy and therefore a lower limit for the total energy input.

Comparing the two lower limits, we find that the average non-thermal energy input is at least of the same order as the change in thermal energy i.e. $\Delta E_{\text {non_th }} \geq \Delta E_{\text {th }}$ in agreement with the prediction of the ITTT model. For a lower electron cut-off energy, the non-thermal energy input could be up to an order of magnitude higher.

\section{Discussion}

The ITTT model as presented by Wheatland \& Melrose (1995) predicts a critical electron energy $E_{\mathrm{c}}$ marking the transition between thin- and thick target emission. This energy is likewise the intersection energy $E_{\text {intersect }}$ between the non-thermal spectra of the coronal and footpoint sources. How can it then be explained in the frame of the ITTT model that in events 2 and 4 the estimated $E_{\mathrm{c}}$ is significantly lower than the observed $E_{\text {intersect }}$ ?

$E_{\mathrm{c}}$ as calculated here may be a lower limit. Including collisional deflections of the electrons within the coronal target rather than just energy-loss, the electron paths would become longer and the critical energy for thin target emission higher. This would increase $E_{\mathrm{c}}$ and could bring it up towards the observed $E_{\text {intersect }}$. In that case however, one would expect to observe a break at $E_{\text {intersect. }}$ This is not the case. Further, the difference $\gamma_{\mathrm{cs}}^{2}-\gamma_{\mathrm{fp}}^{2}$ is higher than 2 in both of these events, making this explanation even less likely.

An attractive possibility to interpret both, the absence of a break and the larger difference in spectral index is noncollisional energy loss of the electrons in the loop due to the electric field caused by the return current. This would result in a harder and fainter footpoint spectrum, shifting $E_{\text {intersect }}$ to energies higher than $E_{\mathrm{c}}$. The expected footpoint spectrum in the case of electrons loosing $15 \mathrm{keV}$ of energy in the loop is indicated in Fig. 1.

The remaining discrepancy then is the difference in spectral index $\gamma_{\mathrm{cs}}^{2}-\gamma_{\mathrm{fp}}^{2}$ being smaller than two in events 1 and 3. Such a difference could result where the targets cannot be clearly separated into thin and thick, for example if the sources are close as is the case in those events. Part of the emission selected for the footpoints could originate from the coronal source, giving a mixed thin-thick target spectrum for the footpoints. More observations are necessary to confirm this explanation.

An estimate of the non-thermal energy input into the coronal source compared to the change of the thermal energy content in the coronal source shows that collisions as manifest in hard Xray emission may account for the observed heating of the coronal source. Depending on the electron cut-off energy, the amount of energy deposited in the target might even exceed the observed thermal energy. However, we did not consider cooling of the coronal source due to thermal conduction or radiation. Such effects could lead to a thermal energy input larger than observed.

\section{Conclusions}

We showed that a simple thin-thick target model as the one proposed by Wheatland \& Melrose (1995) cannot explain all observations made with RHESSI. Modifications of the model are necessary, the simplest being the consideration of the electric field due to the return current. In the absence of a balancing ion flux, such a return current is predicted from basic physics in view of footpoint sources.

Acknowledgements. RHESSI data analysis at ETH Zürich is supported by ETH grant TH-1/04-2 and the Swiss National Science Foundation (grant 20-105366). We thank Paolo Grigis for helpful comments and discussions.

\section{References}

Battaglia, M., \& Benz, A. O. 2006, A\&A, 456, 751 Feldman, U., Hiei, E., Phillips, K. J. H., Brown, C. M., \& Lang, J. 1994, ApJ, 421,843

Fletcher, L., \& Martens, P. C. H. 1998, ApJ, 505, 418

Hoyng, P., Duijveman, A., Machado, M. E., et al. 1981, ApJ, 246, L155

Lin, R. P., Dennis, B. R., Hurford, G. J., et al. 2002, Sol. Phys., 210, 3

Masuda, S., Kosugi, T., Hara, H., et al. 1994, Nature, 371, 495

Metcalf, T. R., \& Alexander, D. 1999, ApJ, 522, 1108

Saint-Hilaire, P., \& Benz, A. O. 2005, A\&A, 435, 743

Veronig, A. M., \& Brown, J. C. 2004, ApJ, 603, L117

Wheatland, M. S., \& Melrose, D. B. 1995, Sol. Phys., 185, 283 